\title{
Wireless Power Transfer to a Microaerial Vehicle with a Microwave Active Phased Array
}

\author{
Shotaro Nako, ${ }^{1}$ Kenta Okuda, ${ }^{1}$ Kengo Miyashiro, ${ }^{2}$ \\ Kimiya Komurasaki, ${ }^{2}$ and Hiroyuki Koizumi ${ }^{3}$ \\ ${ }^{1}$ Department of Aeronautics and Astronautics, The University of Tokyo, Bunkyo-ku, Tokyo 113-8656, Japan \\ ${ }^{2}$ Department of Advanced Energy, The University of Tokyo, Kashiwa-shi, Chiba 277-8561, Japan \\ ${ }^{3}$ Research Center for Advanced Science and Technology, The University of Tokyo, Meguro-ku, Tokyo 153-8904, Japan \\ Correspondence should be addressed to Shotaro Nako; s.nako@al.t.u-tokyo.ac.jp \\ and Kimiya Komurasaki; komurasaki@k.u-tokyo.ac.jp
}

Received 27 December 2013; Revised 31 March 2014; Accepted 3 April 2014; Published 19 May 2014

Academic Editor: Chung-Liang Chang

Copyright (C) 2014 Shotaro Nako et al. This is an open access article distributed under the Creative Commons Attribution License, which permits unrestricted use, distribution, and reproduction in any medium, provided the original work is properly cited.

A wireless power transfer system using a microwave active phased array was developed. In the system, power is transferred to a circling microaerial vehicle (MAV) by a microwave beam of $5.8 \mathrm{GHz}$, which is formed and directed to the MAV using an active phased array antenna. The MAV is expected to support observation of areas that humans cannot reach. The power beam is formed by the phased array with eight antenna elements. Input power is about $5.6 \mathrm{~W}$. The peak power density at $1,500 \mathrm{~mm}$ altitude was $2.63 \mathrm{~mW} / \mathrm{cm}^{2}$. The power is sent to a circling MAV. Therefore, the transfer beam should be polarized circularly to achieve a constant power supply independent of its yaw angle. To minimize the polarization loss, a sequentially routed antenna (SRA) was applied to the transmitter antenna. Results show that the axial ratio of $0.440 \mathrm{~dB}$ was accomplished and that power fluctuation was kept below $1 \%$.

\section{Introduction}

Technologies to supply power wirelessly are being studied intensively around the world. They are anticipated to eliminate restrictions on power supply cables from electric devices. One method, using microwave beam radiation, has attracted attention in applications for more than several meters of distance transfer because of its small attenuation according to distance. It has been developed mainly with emphasis on use in the aerospace field, providing power transfer from a solar power satellite to the ground and from the ground to an aircraft flying through the stratosphere. Details are reported in the literature $[1,2]$.

For this study, the method was assumed to be applied to power transfer to an unmanned microaerial vehicle (MAV) equipped with cameras, microphones, and other sensors. It is useful for observing areas where humans can reach only with great difficulty, such as an area struck by a disaster. In this application, the MAV is free from a landing for charging its batteries or for refueling. The system presents the possibility of lengthy operation and avoids dangers of manual landing.

The flow of wireless power transfer by microwave is as follows. First, a pilot signal of $2.45 \mathrm{GHz}$ radiated from an antenna attached to a MAV is detected by two pairs of tracking antennas each of which are provided for $x$ and $y$ directions. The MAV location is calculated from phase differences between each of the pilot signals detected by the tracking antennas. Secondly, a phase to be given to each transmission antenna forming a phased array is calculated to direct a transfer beam of $5.8 \mathrm{GHz}$ toward the MAV. Finally, rectennas attached to the MAV receive the beam and convert it to DC power. An overview of this system is presented in Figure 1.

The system described above is divided into three subsystems, a tracking, a power-transmission, and a powerreceiving system. In this paper, a power-transmission system is developed. Several reports provide details related to other systems $[3,4]$. 


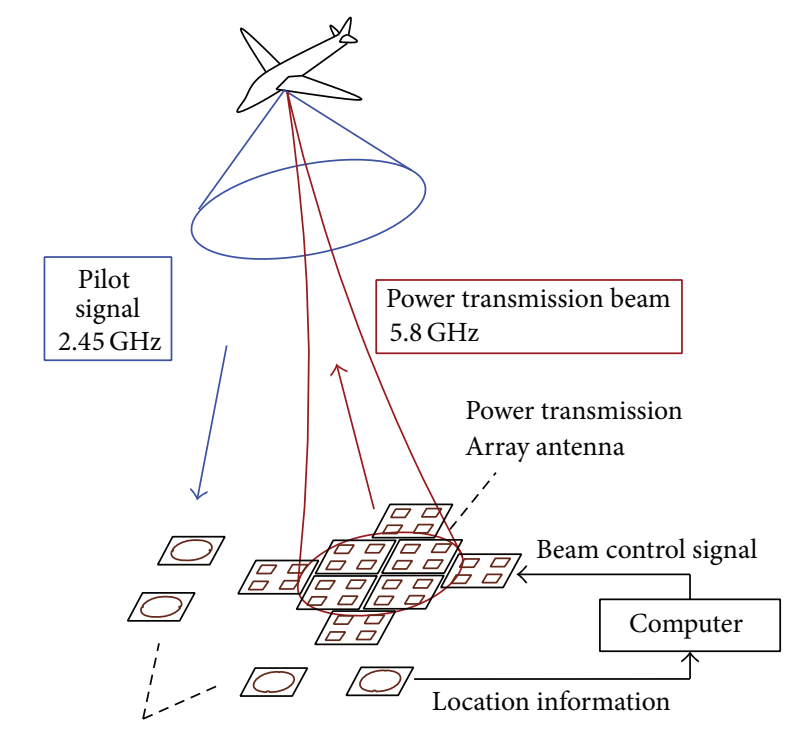

Tracking antenna

FIgURE 1: Overview of wireless power transfer to MAV.

\section{Power-Transmission System}

The transmitting antenna is expected to have a steering mechanism to direct the microwave beam toward the MAV. Because beam steering by phased array antennas is more responsive and accurate than mechanical beam steering, it is suitable for this system in which a target MAV is circling quickly. For the study described in this paper, a phased array antenna is adopted as a transmitting antenna.

A frequency of $5.8 \mathrm{GHz}$ in the industrial, scientific, and medical (ISM) radio bands is used for a transfer beam in this system, while a choice of higher frequency (e.g., $10 \mathrm{GHz}$ [5]) can downsize a whole system.

As the MAV is circling, its yaw angle changes constantly. Then, to minimize the polarization loss that occurs during circling, a transfer wave should be a circular polarized wave. The received power is constantly at the highest value without depending on the yaw angle of the MAV, where the receiving antenna is attached, if the transfer wave is circular polarized and the receiving antenna is for circular polarization. Then, a circular polarization patch antenna is adopted for elements of the phased array antenna. The patch antenna is small, lightweight and can be manufactured at a low cost. In addition, it can make the antenna separation of the phased array small. It engenders a small side lobe especially when the microwave beam is steered widely.

It is difficult to manufacture a completely circular polarized wave patch antenna because its axial ratio depends strongly upon the working accuracy of the milling machine. Therefore, a sequential rotated antenna (SRA) is introduced to the element of the phased array to compensate for a decrease in the axial ratio. The SRA is a subarrayed antenna in which four antenna subelements designed to radiate circularly polarized microwave of $5.8 \mathrm{GHz}$ are attached at four corners of a square. They are set so that their feeding directions are angularly shifted by $90^{\circ}$. Furthermore, the

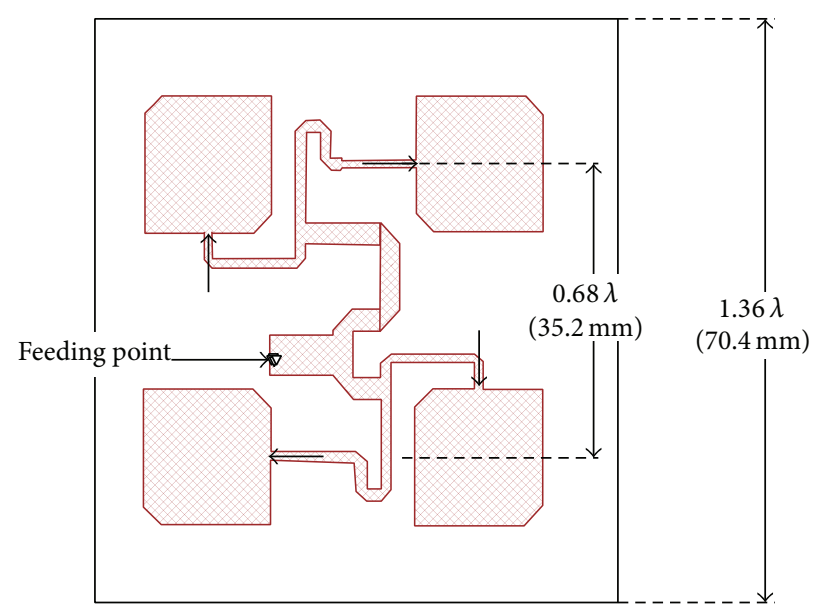

FIGURE 2: SRA formed with a four-antenna subelement.

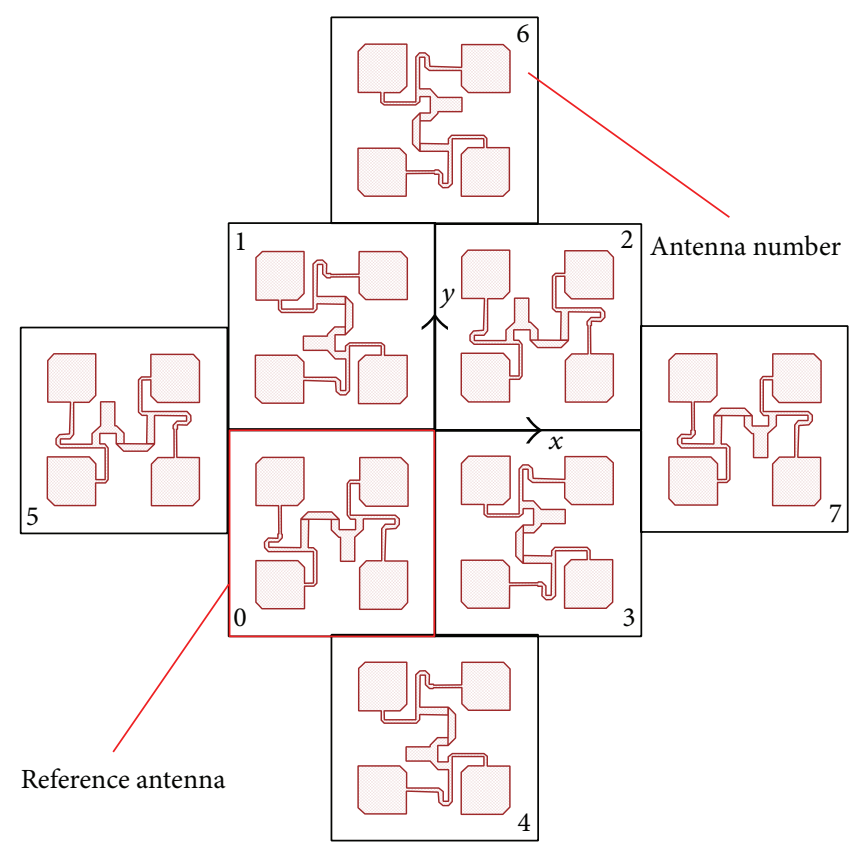

FIGURE 3: Schematic of a seven-element phased array antenna for power transmission.

length of the microstripline feeding of each subelement is adjusted so that microwave beam radiated from each element has the same phase. For that reason, although each subelement might radiate an elliptically polarized wave, power transmission beam, as superposition of those, becomes more circularpolarized. Polarizations of superposed waves are complementary to each other, and it results in generating better axial ratio. The image of SRA is shown in Figure 2. A wave radiated from that is leftward circular polarized. Using it as an element, the power-transmission antenna is formed as shown in Figure 3. The axial ratio of the wave radiated from each element is presented in Table 1. For this report, the axial ratio is defined as the ratio of the minimum to maximum value of electric field intensity in decibel representation. This arrangement of SRA in Figure 3 is an application of SRA 
TABLE 1: Axial ratios for respective SRAs.

\begin{tabular}{lc}
\hline Antenna number & Axial ratio $(\mathrm{dB})$ \\
\hline 0 & 0.472 \\
1 & 0.565 \\
2 & 0.741 \\
3 & 0.438 \\
4 & 0.645 \\
5 & 0.575 \\
6 & 0.670 \\
7 & 0.501 \\
\hline
\end{tabular}

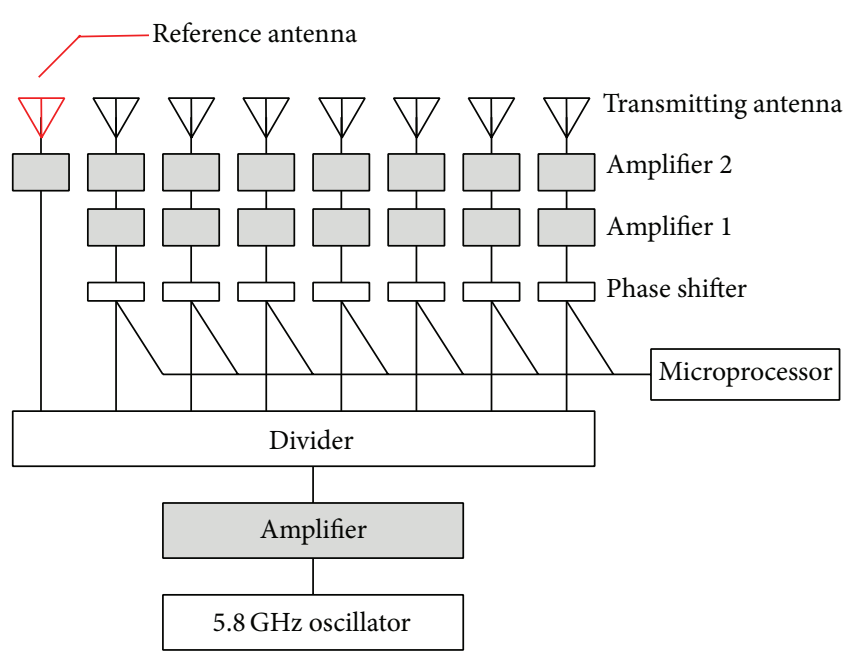

FIGURE 4: Block diagram of power transfer system.

theory. The directions of SRAs are rotated sequentially, which would also engender a high axial ratio. Details of SRAs have been reported [6].

Using this antenna, a transmission system is formed as portrayed in Figure 4. First, the oscillator generates master microwaves of $5.8 \mathrm{GHz}$. Then the divider divides it into eight sets. One is defined as the reference. The phases of the remaining seven sets are shifted by six-bit phase shifters. Then, they are amplified to compensate the attenuation of phase shifters. Finally, the microwaves are amplified once again immediately before the antennas and are radiated from them. The power radiated from the power-transmission system is measured using a power meter immediately before the antenna. It is about $0.7 \mathrm{~W}$ for each element. The total power is $5.6 \mathrm{~W}$.

\section{Results and Discussion}

3.1. Calculation of Transfer Beam Power Density. The twodimensional distribution of power radiated from the transmission antenna is calculated theoretically [7] and compared with the experiment result. It is calculated in the plane of $1,500 \mathrm{~mm}$ from the antenna when the beam steering angles are $0,9.0$, and $12.7 \mathrm{deg}$.
TABLE 2: Computed beam specifications.

\begin{tabular}{lccc}
\hline Steering angle, deg & 0 & 9.0 & 12.7 \\
Peak power density, $\mathrm{mW} / \mathrm{cm}^{2}$ & 2.63 & 2.27 & 1.91 \\
Error of the steering angle, deg & 0.00 & 1.12 & 1.44 \\
\hline
\end{tabular}

TABLE 3: Measured beam specifications.

\begin{tabular}{lcc}
\hline Steering angle, deg & 0 & 9.0 \\
Peak power density, $\mathrm{mW} / \mathrm{cm}^{2}$ & 2.54 & 2.08 \\
Error of the steering angle, deg & 1.15 & 1.12 \\
\hline
\end{tabular}

The calculation result is portrayed in Figure 5. The beam specifications are presented in Table 2. The design maximum steering angle, where the peak of the main lobe matches that of side lobe, is $26 \mathrm{deg}$.

3.2. Measurement Experiment of Transfer Beam Power Density. The two-dimensional distribution of the power radiated from the transmission antenna can be measured. The power distribution is depicted in Figure 6.

The measured beam specifications are presented in Table 3. The peak power density is $2.54 \mathrm{~mW} / \mathrm{cm}^{2}$ for the 0 deg steered case, and it is $2.08 \mathrm{~mW} / \mathrm{cm}^{2}$ for the 9.0 deg steered case. The errors from the theoretical values are, respectively, as small as $2 \%$ and $8 \%$. The error of the peak position from the aimed position is $30 \mathrm{~mm}$ for both 0 and 9.0 deg steered cases. Furthermore, the reduction attributable to steering is $18 \%$. The not-smooth curve surface results from the axial ratio, which differs depending on the position.

3.3. Axial Ratio. At the same time, the axial ratio is also measured. It is derived from the power received by a patch antenna for linear polarization, which is rotated in $10 \mathrm{deg}$ steps from 0 deg to $180 \mathrm{deg}$. Measurements are conducted when the beam is not steered. The power-receiving antenna is located immediately above the transmitting antenna, in the plane at the height of $1,500 \mathrm{~mm}$.

Results show that the axial ratio of microwave beam radiated from the whole transmission antenna is $0.440 \mathrm{~dB}$. This value is smaller than the average of the axial ratio of the SRA elements, $0.575 \mathrm{~dB}$. Furthermore, it is also high compared with that of the non-SRA system described in an earlier report [8]. Moreover, it shows that the introduction of SRA improves the axial ratio. Assuming that a powerreceiving antenna attached to a MVA has the same axial ratio as the transmission system, the power fluctuation according to the yaw angle is less than $1 \%$.

3.4. Quality of Beam Forming. The beam forming specifications are calculated numerically as presented in Table 4 when the beam is not steered. For comparison, that for transmission antenna formed with five antenna elements, shown in Figure 7, is also contained. Beam quality is evaluated using $M^{2}$. The beam diameter is defined as the beam area diameter that has power density higher than $1 / e^{2}$ of the 


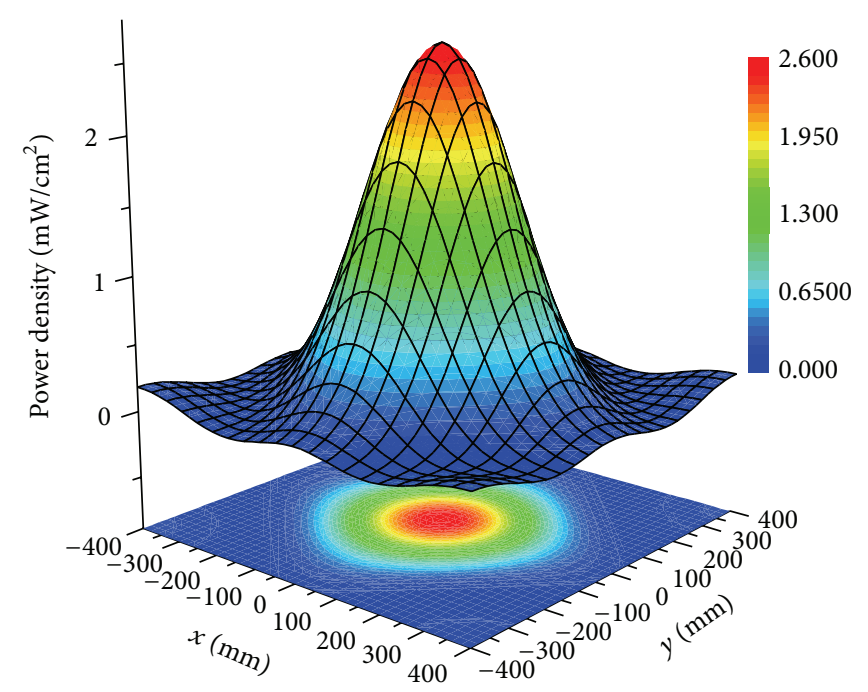

(a) 0 deg steered; peak is at $(0,0)$

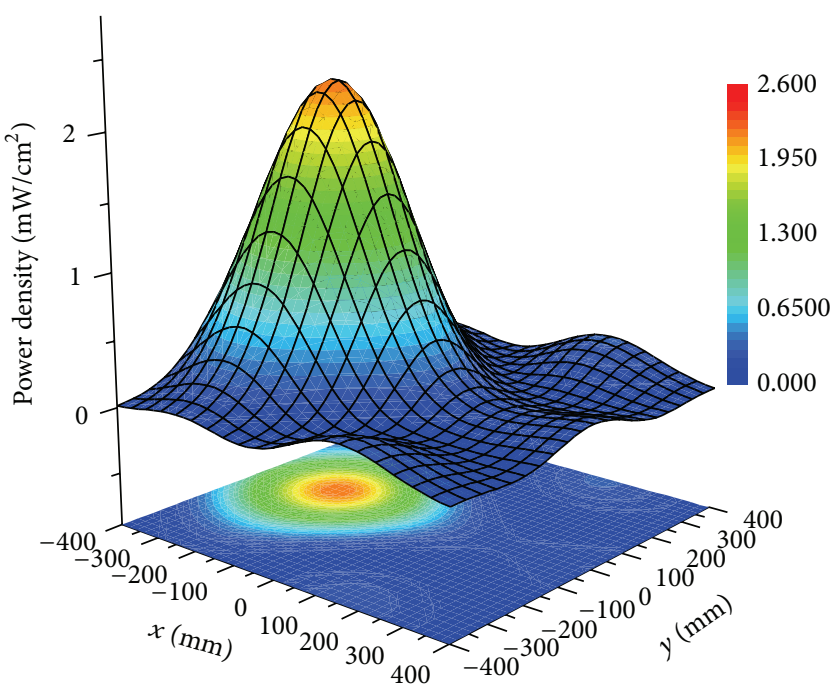

(b) 9.0 deg steered; peak is at $(-240,0)$

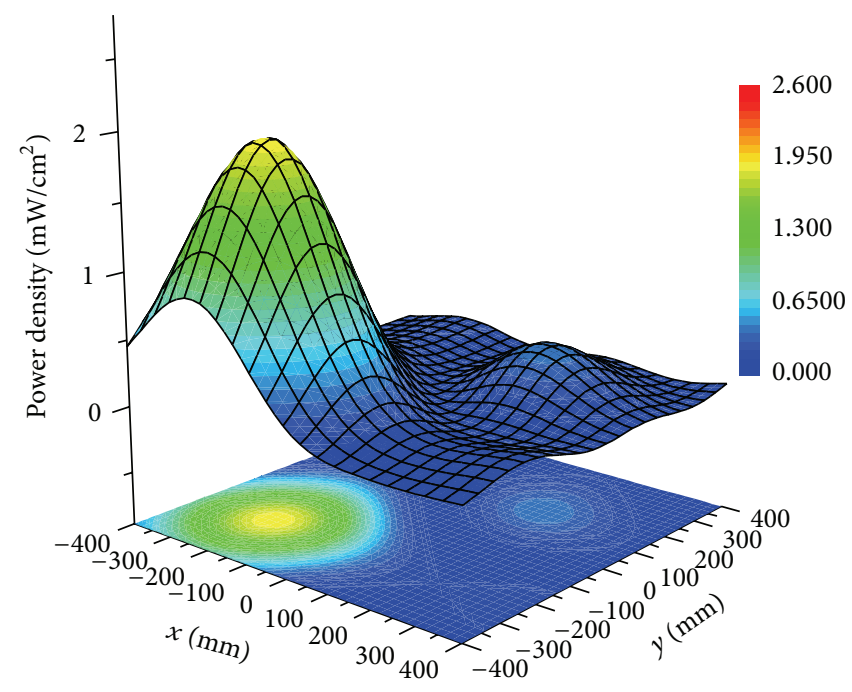

(c) 12.7 deg steered; peak is at $(-240,-240)$

FIgURE 5: Computed power density distribution.

peak in the plane at $1,500 \mathrm{~mm}$ height. Because the effective opening area of receiving antennas and MAV's wing area where they are attached are limited, it is important to suppress the spread of the transfer beam to transmit power at high efficiency. Consequently, the beam diameter for the eightelement antenna system is smaller than that for a five-element antenna system. Similarly, the divergence angle for the eightelement antenna system is expected less than that for a fiveelement antenna system by about $3 \mathrm{deg}$.

\section{Conclusion}

A power-transmission system was constructed for wireless power transfer to a microaerial vehicle (MAV), with beam forming and steering by a phased array having eight antenna elements. Sequentially rotated antennas (SRAs) were used as elements of the phased array. Results show that an axial ratio
TABle 4: Specifications of beam forming.

\begin{tabular}{lcc}
\hline & 8 antennas & 5 antennas \\
\hline Beam aperture diameter, m & 0.550 & 0.670 \\
Beam quality, $M^{2}$ & 1.56 & 2.46 \\
Divergence angle, deg & 10.4 & 12.9 \\
\hline
\end{tabular}

of $0.440 \mathrm{~dB}$ was accomplished and that power fluctuation according to the variation of yaw angle was kept below $1 \%$.

The power distribution of the transfer beam was measured. The peak power density at $1,500 \mathrm{~mm}$ altitude was $2.63 \mathrm{~mW} / \mathrm{cm}^{2}$ with $5.6 \mathrm{~W}$ of total input power. Measured peak power is $98 \%$ of the calculated value. The beam aperture was $0.55 \mathrm{~m}$ in diameter and the beam quality $M^{2}$ was 1.56 , both of which would be small enough to project the main lobe onto a circling MAV. 


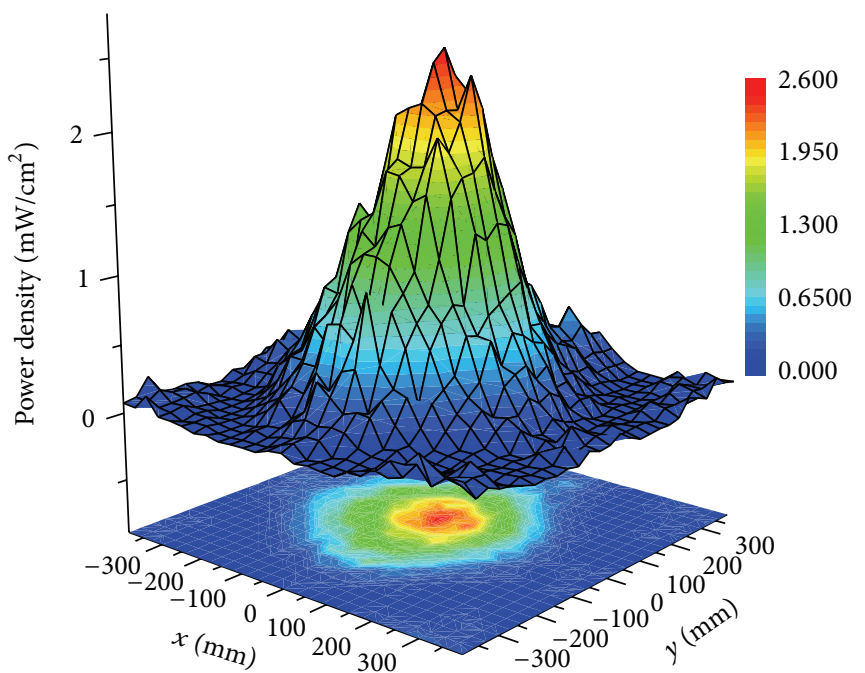

(a) 0 deg steered

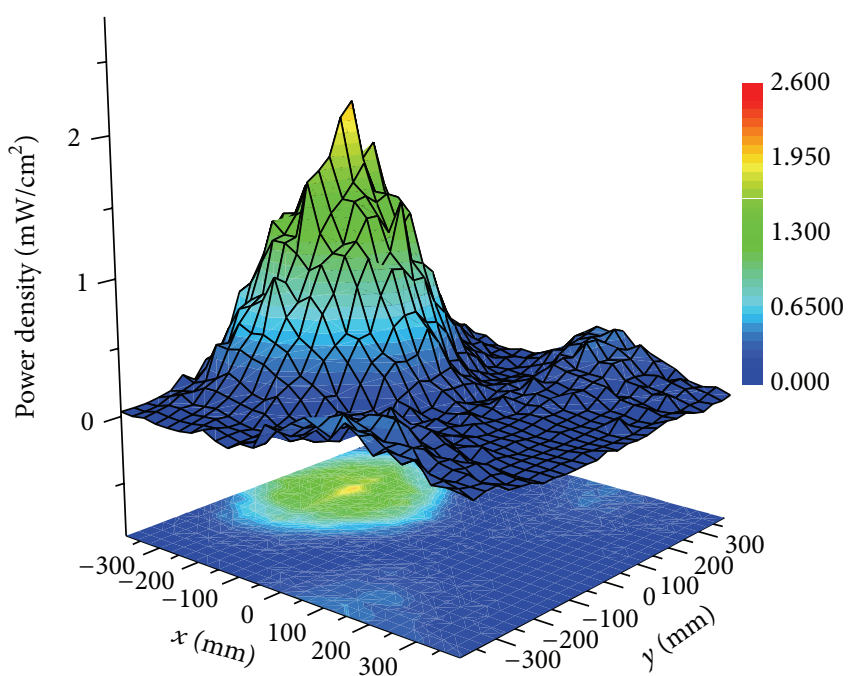

(b) 9.0 deg steered

FIGURE 6: Measured power density distribution.

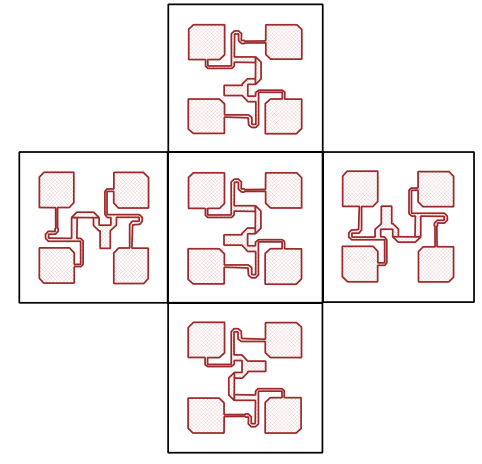

FIGURE 7: Five-element transmission antenna for comparison.
[5] L. H. Toh, A follow-up study on wireless power transmission for unmanned air vehicles [M.S. thesis], Naval Postgraduate School, 2007.

[6] T. Teshirogi, M. Tanaka, and N. Takahashi, "Reduction of mutual coupling effects by sequentially rotated array techniques and its application to phased arrays," Technical Report of IEICE A P96-23, 1996.

[7] M. Haneish, K. Hirasawa, and Y. Suzuki, Small and Planar Antennas, IEICE, 1996.

[8] A. Oda, H. Sawahara, M. Ishiba et al., "Microwave wireless power supply system to a micro aerial vehicle," Journal of The Japan Society for Aeronautical and Space Sciences, vol. 59, no. 691, pp. 222-227, 2011.

\section{Conflict of Interests}

The authors declare that there is no conflict of interests regarding the publication of this paper.

\section{References}

[1] T. W. R. East, "Self-steering array for the SHARP microwavepowered aircraft," IEEE Transactions on Antennas and Propagation, vol. 40, no. 12, pp. 1565-1567, 1992.

[2] N. Shinohara and S. Kawasaki, "Recent wireless power transmission technologies in japan for space solar power station/satellite," in Proceedings of the 2009 IEEE Radio and Wireless Symposium (RWS '09), pp. 13-15, January 2009.

[3] E. Shimane, S. Komatsu, K. Komurasaki, and Y. Arakawa, "AMAV flight using microwave power supply," in Proceedings of the 46th AIAA Aerospace Sciences Meeting and Exhibit, Reno, Nev, USA, January 2008.

[4] S. Komatsu, K. Katsunaga, R. Ozawa, K. Komurasaki, and Y. Arakawa, "Power transmission to a Micro Aerial Vehicle," AIAA Paper 2007-1003, 2007. 

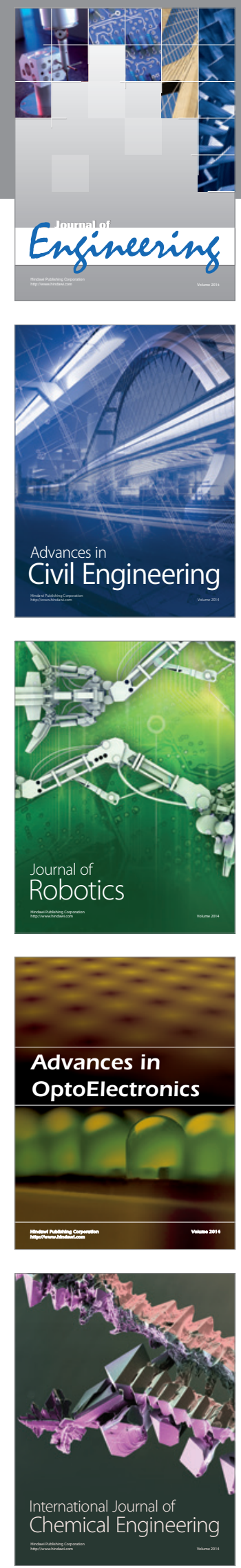

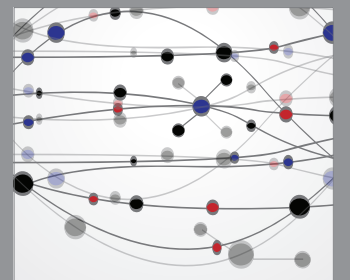

The Scientific World Journal
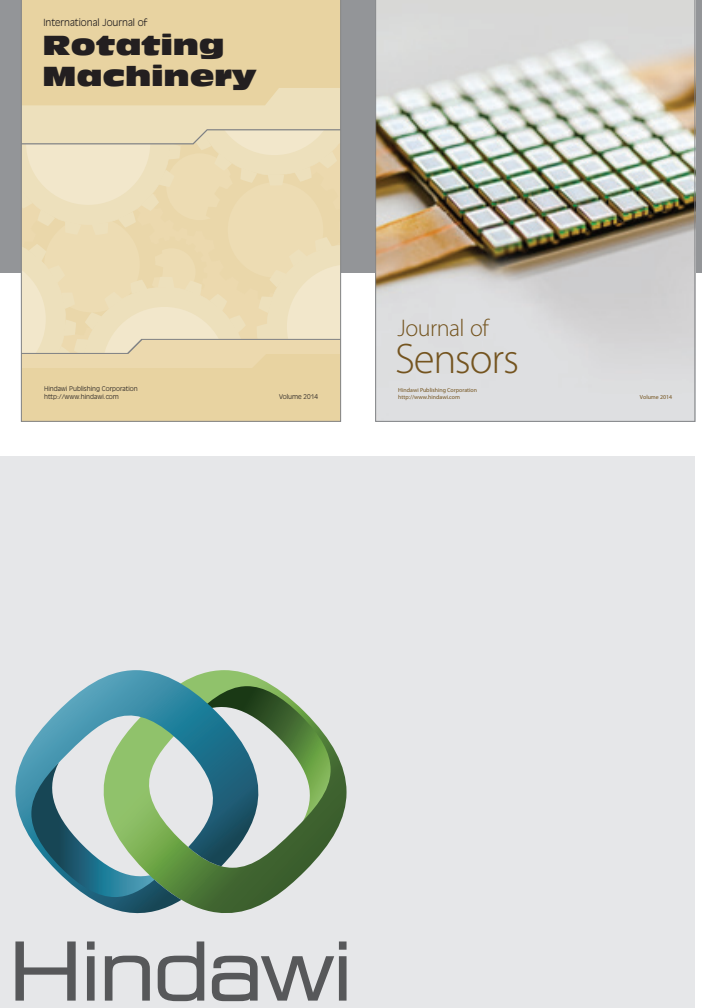

Submit your manuscripts at http://www.hindawi.com
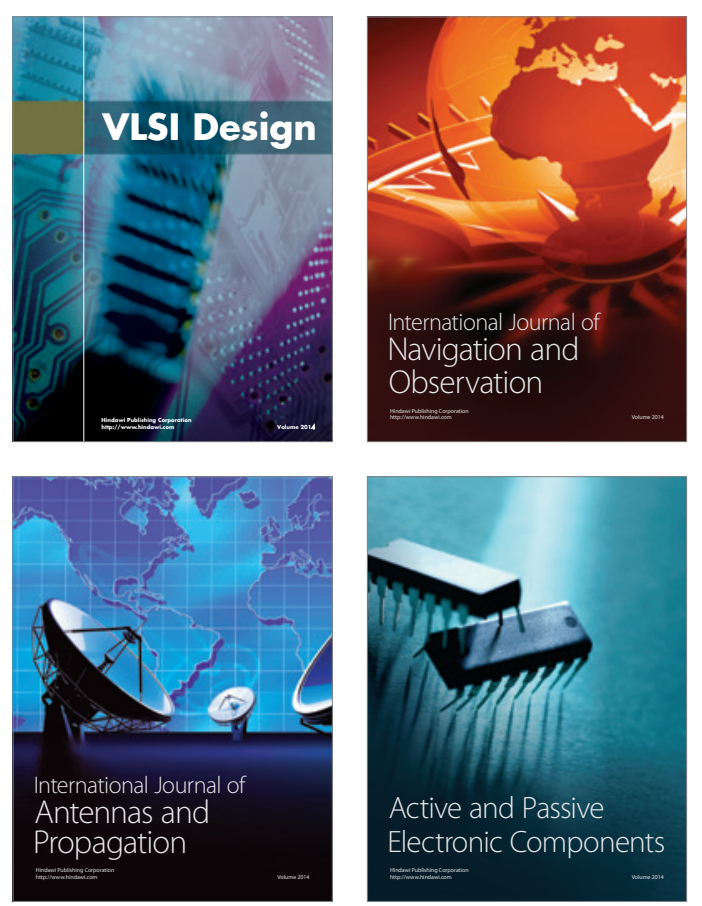
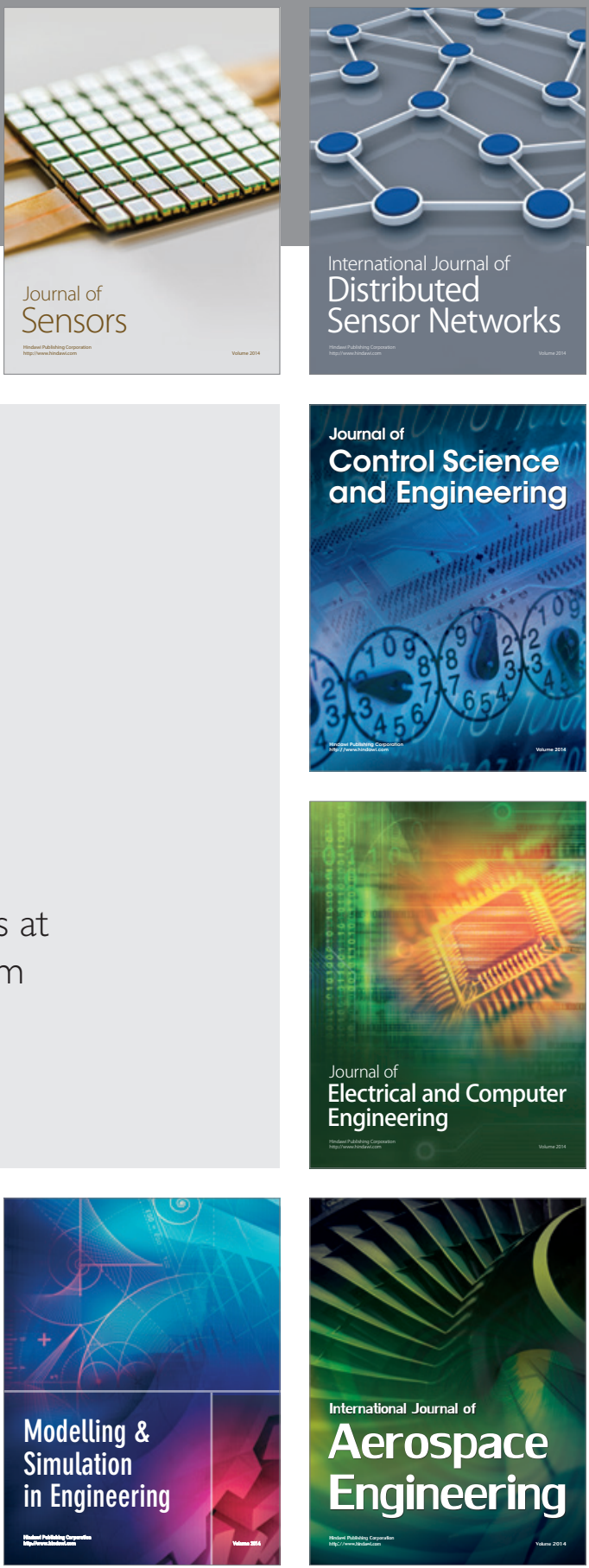

Journal of

Control Science

and Engineering
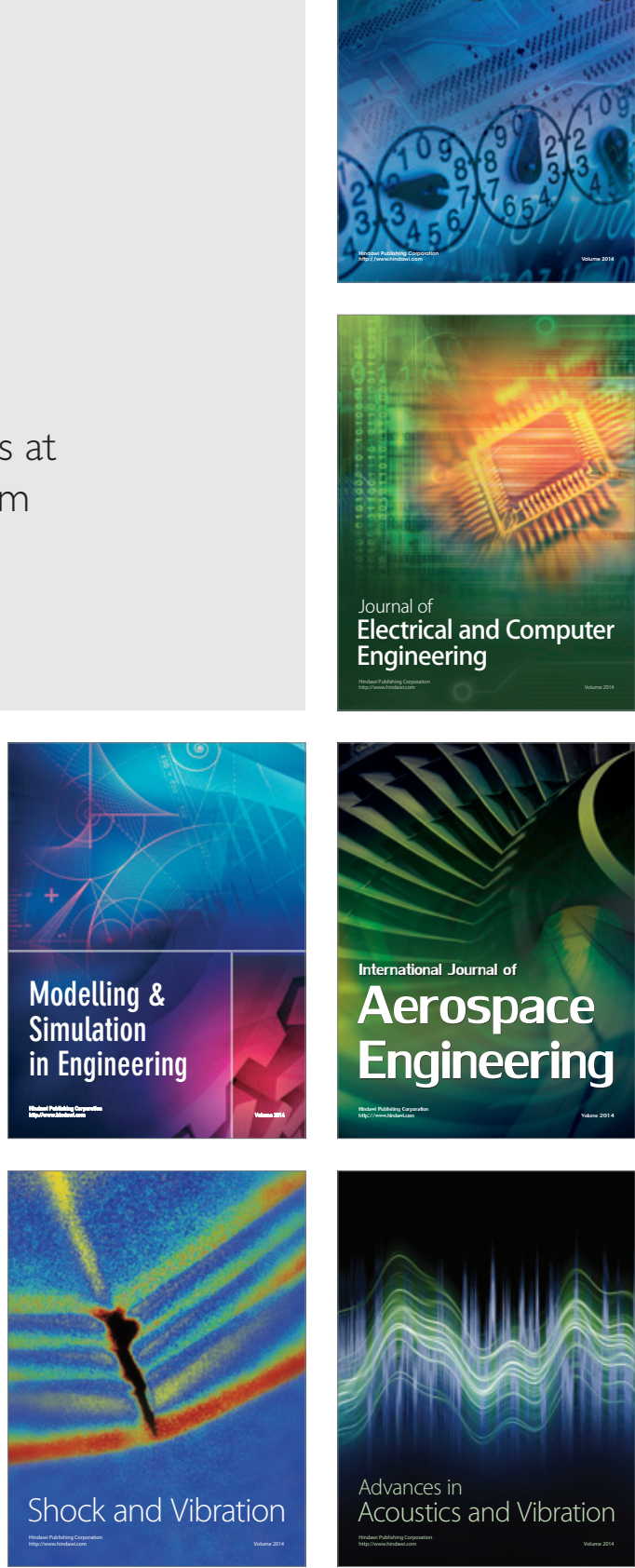